\title{
Hepatobiliary cholesterol transport is not impaired in Abca1-null mice lacking HDL
}

\author{
Albert K. Groen, ${ }^{1}$ Vincent W. Bloks, ${ }^{2}$ Robert H.J. Bandsma, ${ }^{2}$ Roelof Ottenhoff, ${ }^{1}$ \\ Giovanna Chimini, ${ }^{3}$ and Folkert Kuipers ${ }^{2}$
}

${ }^{1}$ Department of Gastroenterology, Academic Medical Center, Amsterdam, The Netherlands ${ }^{2}$ Department of Pediatrics, University Hospital Groningen, Groningen, The Netherlands ${ }^{3}$ Centre de Immunologie, INSERM-CNRS, Marseille, France

Address correspondence to: Albert K. Groen, Department of Gastroenterology, F0-222, Academic Medical Center, Meibergdreef 9, 1105 AZ Amsterdam, The Netherlands.

Phone: 31-205664174; Fax: 31-205669190; E-mail: a.k.groen@amc.uva.nl.

Received for publication February 8, 2001, and accepted in revised form July 30, 2001.

\begin{abstract}
The $\mathrm{ABC}$ transporter $\mathrm{ABCA1}$ regulates HDL levels and is considered to control the first step of reverse cholesterol transport from the periphery to the liver. To test this concept, we studied the effect of ABCA1 deficiency on hepatic metabolism and hepatobiliary flux of cholesterol in mice. Hepatic lipid contents and biliary secretion rates were determined in $A b c a 1^{-/-}, A b c a 1^{+/-}$, and $A b c a 1^{+/+}$mice with a DBA background that were fed either standard chow or a high-fat, high-cholesterol diet. Hepatic cholesterol and phospholipid contents in $\mathrm{Abca1}^{-/-}$mice were indistinguishable from those in $\mathrm{Abca1^{+/- }}$ and $A b c a 1^{+/+}$mice on both diets. In spite of the absence of HDL, biliary secretion rates of cholesterol, bile salts, and phospholipid were unimpaired in $A b c a 1^{-/-}$mice. Neither the hepatic expression levels of genes controlling key steps in cholesterol metabolism nor the contribution of de novo synthesis to biliary cholesterol and bile salts were affected by $A b c a$ genotype. Finally, fecal excretion of neutral and acidic sterols was similar in all groups. We conclude that plasma HDL levels and ABCA1 activity do not control net cholesterol transport from the periphery via the liver into the bile, indicating that the importance of HDL in reverse cholesterol transport requires re-evaluation.
\end{abstract}

J. Clin. Invest. 108:843-850 (2001). DOI:10.1172/JCI200112473.

\section{Introduction}

It is generally assumed that HDL mediates transport of excess cholesterol from peripheral tissue to the liver, a process called reverse or centripetal cholesterol transport (1). According to current knowledge, the liver is the only organ capable of removing excess cholesterol from the body via its excretion into bile, either in the form of free cholesterol or after its conversion to bile salts (2). Reverse cholesterol transport originates at extrahepatic organs and peripheral macrophages where cholesterol is taken up by nascent HDL, possibly together with phospholipid (3). Scavenger receptor-BI (SR-BI) was demonstrated to be the main receptor involved in uptake of cholesterol(ester) and phospholipid from HDL (4-6) in the liver.

The crucial role of this receptor was highlighted in studies with transgenic and knockout animals (5, 7-9). Transient overexpression led to a very strong decrease of plasma HDL, whereas HDL was considerably increased in SR-BI-null mice $(5,7)$. Although SR-BI can accommodate bidirectional cholesterol flux (10), its role in mediating efflux of cholesterol from peripheral tissues is probably less important (11). Patients with Tangier disease or familial HDL deficiency (FHD) with very low levels of plasma HDL accumulate cholesterol(ester), particularly in tissues that are rich in macrophages (12). Tangier disease and FHD are both caused by mutations in the gene encoding ABCA1, a member of the large superfamily of ATP binding cassette transporters (13-15). Fibroblasts derived from patients with Tangier disease or FHD are defective in the efflux of cholesterol and phospholipid towards apoA-I, and partially defective in efflux towards HDL (16-21).

The function of ABCA1 is not confined to transport of cholesterol and phospholipid: the protein is also functional in phagocytosis $(22,23)$. Macrophages from $A b c a 1^{-/-}$mice were shown to be impaired in engulfment of apoptotic bodies $(22,23)$. In addition, the knockout mice showed defects in platelet aggregation caused by a defect in exposing phosphatidylserine at the outer leaflet of the platelet plasma membrane (23). This led Hamon et al. (23) to conclude that ABCA1 may be involved in controlling phospholipid asymmetry in the plasma membrane. Overexpression of the protein in macrophages induced changes in cell morphology that may be caused by changes in membrane composition (24). In fact, recent work (25) indicates that ABCA1induced modification of lipid distribution in the membrane generates a microenvironment that is required for docking of apoA-I at the cell surface. Apart from macrophages, ABCA1 is expressed in other tissues, including liver and small intestine (26). The function of the protein in the liver has, to the best of our knowledge, not yet been studied. 
Intestinal $\mathrm{ABCA} 1$ has been suggested to be involved in efflux of cholesterol from intestinal epithelial cells into the lumen, thereby influencing the efficiency of intestinal cholesterol absorption (27). However, the exact localization of ABCA1 in the intestinal epithelial cells has not yet been reported. In this study we investigated the consequences of absence of HDL caused by ABCA1 deficiency on hepatic cholesterol metabolism and hepatobiliary cholesterol flux in mice. HDL has been suggested, in rodents as well as in humans, to be an important carrier of bile-destined cholesterol and phospholipid (28-30). Since the $A b c a 1^{-/-}$mice lack HDL $(31,32)$, and therefore, according to current knowledge, should not be able to perform active reverse cholesterol transport, we expected these animals to show significant alterations in biliary cholesterol secretion, possibly (partly) compensated for by changes in hepatic cholesterol metabolism. Surprisingly, no alteration in any of the parameters of hepatic cholesterol metabolism was found, suggesting that current concepts of regulation of body cholesterol homeostasis may require revision.

\section{Methods}

Animals. Abca1 $1^{-/}$and $A b c a 1^{+/-}$mice, generated as described elsewhere (23), and wild-type mice with a DBA/ 1 background, 3-6 months of age were obtained from Iffa Credo (L'Arbresle, France). Experimental protocols were approved by the Ethics Committees for Animal Experiments at the University of Groningen and the University of Amsterdam. Because homozygous knockout mice are difficult to breed (31), the animal supply was limited, and both male and female mice were used. Despite the fact that no sex-related differences were observed, sex distribution was held constant in the separate sets of experiments. Animals were fed for 2 weeks ad libitum with either lab chow (RMH-B; Hope Farms BV, Woerden, The Netherlands) or an atherogenic high-cholesterol diet, containing $1 \%$ (wt/wt) cholesterol, $0.5 \%$ cholate, $15 \%$ cocoa butter, $1 \%$ corn oil, $10 \%$ corn starch, $20 \%$ casein, $4.7 \%$ cellulose, and $40 \%$ sucrose (Hope Farms BV). When indicated, chow was supplemented with $\left[1-{ }^{13} \mathrm{C}\right]$ acetate $(20 \mathrm{mg} / \mathrm{g}$ chow) (33) (Isotec Inc., Miamisburg, Ohio, USA) for 10 days prior to bile collection, to allow assessment of the contribution of de novo synthesized cholesterol to biliary cholesterol and bile salts (34).

Experimental procedures. Bile was collected by cannulation of the gallbladder under Hypnorm (fentanyl/fluanisone; $1 \mathrm{ml} / \mathrm{kg})$ and diazepam $(10 \mathrm{mg} / \mathrm{kg})$ anesthesia, using a humidified incubator to maintain body temperature. Bile collection was performed for 30-45 minutes, and production was determined gravimetrically. At the end of the procedure, a large blood sample was taken by cardiac puncture, and the liver and small intestine were rapidly excised. Parts of the liver were frozen in liquid nitrogen for later analyses. Segments of liver tissue and small intestine were fixed in paraformaldehyde for later staining with hematoxylin and eosin. To determine maximal rates of biliary lipid secretion, bile was diverted for 90 minutes to deplete the endogenous bile salt pool. Subsequently, tauroursodeoxycholate (TUDC; Sigma Chemical Co., St. Louis, Missouri, USA) dissolved in PBS was infused into the tail vein at stepwise-increasing rates from 600 to 2400 $\mathrm{nmol} / \mathrm{min}$ per $100 \mathrm{~g}$ body weight. Bile was collected in 10-minute fractions and analyzed for bile salt and lipid content. To assess kinetics of biliary excretion of plasma-derived cholesterol, extruded neutral liposomes (average size $80 \mathrm{~nm}$ ) composed of egg phosphatidylcholine and cholesterol (60:40) containing $\left[{ }^{3} \mathrm{H}\right]$ cholesteryloleylether and $\left[4-{ }^{14} \mathrm{C}\right]$ cholesterol (both from NEN Life Science Products, Boston, Massachusetts, USA) as radiolabel were intravenously injected via the penile vein in wild-type and $\mathrm{Abca1^{-/- }}$ mice, immediately after creation of a bile fistula. The injected dose was $2 \mu \mathrm{mol}$ total lipid per $100 \mathrm{~g}$ body weight. Bile was collected in 30-minute fractions for 2 hours; thereafter a large blood sample was collected by cardiac puncture and liver and spleen were rapidly excised. Liver and spleen were homogenized in PBS, and ${ }^{3} \mathrm{H}$ and ${ }^{14} \mathrm{C}$ radioactivities were measured in plasma, liver, and spleen by scintillation counting as described (35).

Mass isotopomer distribution analyses. To determine de novo synthesis of cholesterol and bile salts, mice were fed a diet containing $\left[1-{ }^{13} \mathrm{C}\right]$ acetate for 10 days $(20 \mathrm{mg} / \mathrm{g}$ chow ) (33). Animals ate the diet in normal quantities and grew normally. After 10 days, bile samples were taken and frozen. Biliary cholesterol was derivatized using $\mathrm{N}, \mathrm{O}$-bis(trimethylsilyl)trifluoroacetamide with $1 \%$ trimethylchlorosilane, at room temperature (36). Bile salts were derivatized by adding $20 \mu \mathrm{l}$ triethylamine and $100 \mu \mathrm{l}$ of a $10 \%$ pentafluorobenzyl bromide solution in acetonitrile and incubating for 20 minutes at room temperature. After extraction with ethyl acetate, dried samples were derivatized by the addition of $100 \mu \mathrm{N}, \mathrm{O}$-bis-(trimethylsilyl)trifluoroacetamide, pyridine, and trimethylchlorosilane. All analyses were performed on a Finnigan SSQ7000 GC/MS instrument (Finnigan Corp., San Jose, California, USA). For cholesterol, mass fragments with $m / z 368-371$ were analyzed. For cholate, mass fragments with $m / z$ 623-627 were analyzed. The theoretical background of the mass isotopomer distribution analysis (MIDA) technique is described in detail elsewhere (37-39). Briefly, MIDA allows determination of the enrichment of the pool of acetyl CoA precursor units that has entered newly synthesized cholesterol or bile salt molecules during the course of $\left[{ }^{13} \mathrm{C}\right]$ acetate feeding. Through analysis of the isotopomer pattern of these molecules, it becomes possible to determine the fraction of newly synthesized cholesterol and cholate molecules (34).

$R T-P C R$. RNA isolation and cDNA synthesis were done according to Bloks et al. (40). RT-PCR was performed in $50-\mu \mathrm{l}$ preparations using $2 \mu \mathrm{l}$ cDNA, $0.25 \mu \mathrm{l}$ (1.25 U) Taq polymerase, $5.0 \mu \mathrm{l}$ tenfold buffer, $0.75 \mu \mathrm{l}$ dNTP mix (10 mM) (all from Roche Molecular Biochemicals, Mannheim, Germany), 2.0 $\mu$ l DMSO (Merck KGaA, Darmstadt, Germany), and $1.0 \mu \mathrm{l}$ of each primer 
(50 pmol, GIBCO, Life Technologies, Breda, The Netherlands). Primer sequences $\left(5^{\prime}\right.$ to $\left.3^{\prime}\right)$ used were: GGA GCG TGG ACC CTA TGT CT and TAG AGG CTC AGC TGC ATC TT for SRB1 (srb1; GenBank accession no. U37799); CAA TGG CAC GCT GCA GAC CCT G and ATG GCC TTC CTC AGA ACG CGA G for sterol regulatory element binding protein 2 (SREBP2) (srebp2; GenBank accession no. U02031); GAC ACT TAC TAT CTG TAT GAT TG and CTT GGA GAG GTA AAA CTG CCA for HMG-CoA reductase ( $h m g r ;$ GenBank accession no. M29249); GGA GTG CAT CAG CTT GGA CA and GTG ATG CCA TTT GGC CAG TG for LDL receptor (ldlr; GenBank accession no. X64414); and AAC ACC CCA GCC ATG TAC G and ATG TCA CGC ACG ATT TCC C for $\beta$-actin (41) ( $\beta$-actin; GenBank accession no. M12481). The PCR procedure consisted of predenaturation at $95^{\circ} \mathrm{C}$ for 2 minutes, and a final extension period of 5 minutes at $72^{\circ} \mathrm{C}$. Amplification conditions, starting all primer sets with $30 \mathrm{sec}-$ onds at $95^{\circ} \mathrm{C}$, were as follows: annealing for 30 seconds at $62^{\circ} \mathrm{C}$ for srb1 (25 cycles), at $60^{\circ} \mathrm{C}$ for srebp2 (28 cycles), at $56^{\circ} \mathrm{C}$ for $l d l r$ ( 25 cycles), at $55^{\circ} \mathrm{C}$ for $\beta$-actin (20 cycles), and at $52^{\circ} \mathrm{C}$ for $h m g r(23$ cycles), followed by an extension for 30 seconds at $72^{\circ} \mathrm{C}$ for all primer sets. In pilot experiments, cycle numbers were carefully checked to avoid saturation. The PCR products were separated on a $2.5 \%$ agarose gel. The intensity of the ethidium bromide staining was measured with an ImageMaster VDS gel documentation system and ImageMaster 1D Elite software (Pharmacia Biotech AB, Uppsala, Sweden).

Analytical procedures. Plasma total cholesterol, free cholesterol, and triglycerides were measured using commercially available kits according to the manufacturer's instructions (Roche Molecular Biochemicals). Plasma phospholipids were determined using a kit from Wako Chemicals GmbH (Neuss, Germany). Contents of cholesterol, phospholipid, and triglyceride in liver tissue were determined after lipid extraction as described previously (41). Biliary bile salt, cholesterol, and phospholipid concentrations were determined as described previously $(42,43)$. Biliary phospholipid species were analyzed by TLC as described by Smit et al. (44).

Fecal sterol analysis. Abca1 $1^{-/}, \mathrm{Abca1}^{+/-}$, and $A b c a 1^{+/+}$mice were housed individually, and total fecal production during a 1-week period was separated from the wood shavings. Fecal samples were lyophilized and weighed. Aliquots thereof were used for determination of neutral and acidic sterol content by gas liquid chromatographic procedures (42).

Statistical analysis. All results are presented as mean \pm SD for the number of animals indicated. Differences between different Abca1 genotypes were determined by ANOVA and Newman-Keuls post-hoc $t$ test. Level of significance was set at $P<0.05$. Analysis was performed using SPSS for Windows software (SPSS Inc., Chicago, Illinois, USA).

\section{Results}

Table 1 summarizes values for plasma, liver, and biliary lipid concentrations for the groups of mice used in this study. As reported previously $(31,32)$, plasma cholesterol, phospholipid, and triglyceride concentrations were decreased by about $80 \%$ in $A b c a 1^{-/-}$mice compared with wild-type controls. Plasma lipid levels in heterozygotes showed an intermediate decrease. In contrast, hepatic lipid levels were not changed in $\mathrm{Abca1}^{-/-}$or $\mathrm{Abca1^{+/- }}$ mice when compared with wild-type mice. In contrast to expectations, bile flow and basal biliary secretion rates of cholesterol, bile salts, and phospholipids were not impaired in $\mathrm{Abca1}^{-/-}$or $\mathrm{Abca1^{+/- }}$ mice fed the low-cholesterol diet (Table 1). Because we considered the lack of effect on basal biliary lipid secretion very surprising, we next studied the effect of $A b c a 1$ disruption under conditions of maximal biliary lipid output induced by infusion of TUDC. Mice were first depleted from their endogenous pool of bile salt and then infused via the tail vein with increasing doses of TUDC. As shown in Figure 1, there were no differences in bile salt, cholesterol, or phospholipid secretion between $A b c a 1^{-/-}$and wild-type mice during the 90-minute depletion phase. With increasing infusion rates of TUDC, bile salt secretion increased to a similar extent in both wild-type and knockout mice. Also, biliary cholesterol secretion was not impaired and reached the same maximal value in $\mathrm{Abca1}^{-/-}$and wild-type mice. In contrast, phospholipid secretion was somewhat lower in the $A b c a 1^{-/-}$mice during the infusion phase: a slight but significant $(P<0.05)$ decrease of about $20 \%$ in the transport maximum was found. Expression levels of the hepatic phospholipid flippase ABCB4 (Mdr2) were not changed in $\mathrm{Abca1^{-/- }}$ mice, and bile phospholipid was almost exclusively composed of phosphatidylcholine (data not shown), as in wild-type mice.

We found no significant differences in hepatic mRNA levels of the HDL receptor SR-BI, the LDL receptor, HMG-CoA reductase, or SREBP2 between

\section{Table 1}

Plasma, hepatic, and biliary lipid values in $\mathrm{Abca1^{+/+ }}, \mathrm{Abca1^{+/- }}$, and $\mathrm{Abca1^{-/- }}$ mice

\begin{tabular}{|c|c|c|c|}
\hline Genotype & $A b c a 1^{+/+}$ & $A b c a 1^{+/-}$ & $A b c a 1^{-/-}$ \\
\hline Plasma cholesterol (mM) & $1.95 \pm 0.23$ & $1.26 \pm 0.24^{\mathrm{A}}$ & $0.29 \pm 0.08^{\mathrm{AB}}$ \\
\hline Plasma phospholipids (mM) & $2.32 \pm 0.18$ & $1.56 \pm 0.18^{A}$ & $0.38 \pm 0.08^{A B}$ \\
\hline Plasma triglycerides (mM) & $1.18 \pm 0.26$ & $0.82 \pm 0.38$ & $0.31 \pm 0.12^{\mathrm{A}}$ \\
\hline Liver free cholesterol $(\mu \mathrm{mol} / \mathrm{g})$ & $6.2 \pm 0.5$ & $6.5 \pm 0.4$ & $6.9 \pm 0.8$ \\
\hline Liver cholesterol(ester) $(\mu \mathrm{mol} / \mathrm{g})$ & $0.7 \pm 0.2$ & $0.4 \pm 0.1$ & $0.8 \pm 0.3$ \\
\hline Liver phospholipids ( $\mu \mathrm{mol} / \mathrm{g}$ ) & $41.1 \pm 2.9$ & $40.5 \pm 2.7$ & $41.1 \pm 5.2$ \\
\hline Liver triglycerides $(\mu \mathrm{mol} / \mathrm{g})$ & $11.5 \pm 4.4$ & $12.1 \pm 6.0$ & $14.4 \pm 5.3$ \\
\hline $\begin{array}{l}\text { Biliary cholesterol secretion } \\
(\mathrm{nmol} / \mathrm{min} / 100 \mathrm{~g} \mathrm{BW})\end{array}$ & $6.7 \pm 4.8$ & $6.1 \pm 3.4$ & $9.9 \pm 2.6$ \\
\hline $\begin{array}{l}\text { Biliary phospholipid secretion } \\
(\mathrm{nmol} / \mathrm{min} / 100 \mathrm{~g} \mathrm{BW})\end{array}$ & $51 \pm 25$ & $50.8 \pm 17.7$ & $66.9 \pm 13.3$ \\
\hline $\begin{array}{l}\text { Biliary bile salt secretion } \\
(\mathrm{nmol} / \mathrm{min} / 100 \mathrm{~g} \mathrm{BW})\end{array}$ & $411 \pm 298$ & $335 \pm 134$ & $444 \pm 123$ \\
\hline
\end{tabular}

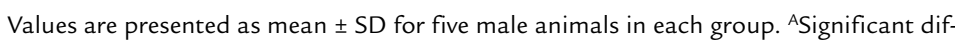
ference when compared with wild-type $\left(A b c a 1^{+/+}\right)$mice. ${ }^{B}$ Significant difference when compared with heterozygous $\left(\mathrm{Abca}^{+/-}\right)$mice. BW, body weight. 


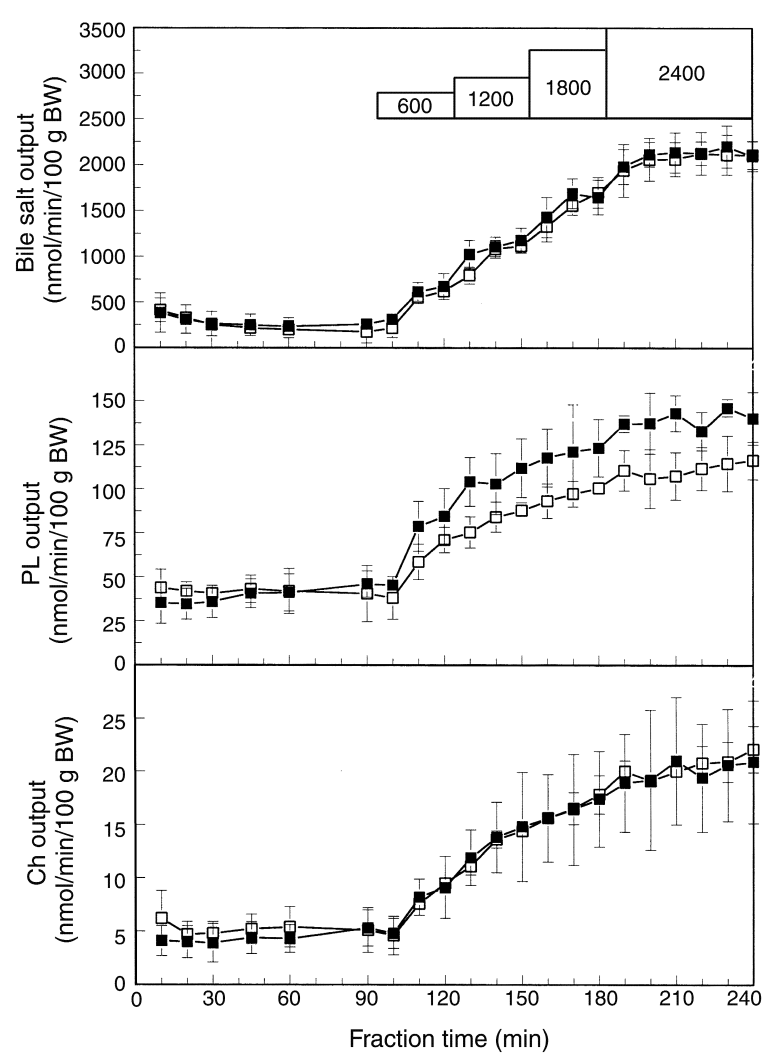

Figure 1

Biliary bile salt and lipid secretion in $A b c a 1^{+/+}$and $A b c a 1^{-/-}$mice during infusion of TUDC. The gallbladders of mice were cannulated and bile was collected for 90 minutes in order to deplete the endogenous bile salt pool. Subsequently, TUDC was infused via the tail vein in a stepwise-increasing fashion, as indicated by the numbers $(\mathrm{nmol} / \mathrm{min} / 100 \mathrm{~g} \mathrm{BW})$ in the figure. Data represent mean \pm SD for four female mice in each group; during the infusion phase there was a significant difference in phospholipid secretion between wild-type (filled boxes) and knockout (open boxes) mice. $P<0.05$ (ANOVA). Ch, cholesterol. PL, phospholipid.

any of the genotypes $\left(A b c a 1^{-/-}, A b c a 1^{+/-}\right.$, and wild-type mice) (Figure 2). This indicates that no major changes in hepatic cholesterol metabolism were induced by ABCA1 deficiency. To evaluate whether or not biliary cholesterol and bile salts were derived from different sources of cholesterol in the bodies of mice with varying HDL levels, the contribution of newly synthesized cholesterol to biliary bile salt and cholesterol secretion was determined by MIDA. The calculated precursor pool enrichments and the fractions of newly synthesized cholesterol and cholate, the major bile salt species in mice, were similar in bile of mice of all three genotypes (Table 3 ). This finding indicates that HDL deficiency does not affect the origin of cholesterol entering the biliary removal pathway in mice fed a low-cholesterol diet. No differences in mRNA levels of HMG-CoA reductase in the distal ileum were found among the three groups of animals (data not shown), indicating unaltered intestinal cholesterol synthesis. In addition, fecal excretion of bile salts and neutral sterols was unchanged by (partial) ABCA1 deficiency, indicating unaltered whole-body cholesterol synthesis (Table 3). To confirm whether in fact the plasmato-bile flux of cholesterol was unchanged in ABCA1deficient mice, we injected mice intravenously with liposomes containing ${ }^{3} \mathrm{H}$-labeled cholesteryloleylether (as a marker that does not metabolize) and ${ }^{14} \mathrm{C}$ labeled free cholesterol. The liposomes were sufficiently small $(\sim 80 \mathrm{~nm})$ to be able to pass the fenestrae in the endothelial cells, allowing direct uptake by the hepatocytes $(35,45)$. At 2 hours after injection, $A b c a 1^{+/+}$and $\mathrm{Abca1}^{-/-}$mice had accumulated about $15 \%$ of the administered cholesteryloleylether in their livers, probably in the lysosomal compartment (35, 45). As shown in Figure 3, accumulation of liposomal free cholesterol in the liver was somewhat lower in $A b c a 1^{-/-}$mice than in $\mathrm{Abca1}^{+/+}$mice. Accordingly, biliary secretion of $\left[{ }^{14} \mathrm{C}\right]$ cholesterol was also slightly lower in the knockouts, but the differences were not statistically significant.

In a last set of experiments, we stressed body cholesterol homeostasis by feeding the mice a high-cholesterol, high-fat diet also containing $0.5 \%$ (wt/wt) cholate. After 2 weeks on the diet, plasma levels of cholesterol were increased by $50 \%, 200 \%$, and $400 \%$ in wildtype, $A b c a 1^{+/-}$, and $A b c a 1^{-/-}$mice, respectively, when compared with animals of the same genotype fed normal chow (Table 4). Hepatic contents of free cholesterol and triglycerides were approximately doubled in mice of all three genotypes after feeding the atherogenic diet. As expected, cholesterol(ester) levels increased massively on the high-cholesterol diet, from less than 1 $\mu \mathrm{mol} / \mathrm{g}$ liver to approximately $50 \mu \mathrm{mol} / \mathrm{g}$ liver. However, this increase was again similar in mice of all genotypes (Table 4). The increased dietary cholesterol load induced a fourfold increase of biliary cholesterol secretion and a doubling of biliary phospholipid and bile salt output. Amazingly, the increases were almost identical in wild-type, $\mathrm{Abca1}^{+/-}$, and $\mathrm{Abca1^{-/- }}$ mice. Histological examination of mouse livers revealed the development of fatty livers in all genotypes fed the atherogenic diet (not shown). In contrast to what has recently been reported for the $A b c a 1^{-/-}$mouse on a C57BL/6 background (46), we observed no alterations in liver or intestinal histology in $\mathrm{Abca1}^{-/-}$(or $\mathrm{Abca1^{+/- }}$ ) mice on the DBA/ 1 background. Liver architecture was completely normal, and no lipid droplets nor other alterations were observed on normal diet.

\section{Table 2}

Daily fecal sterol excretion in $A b c a 1^{+/+}, A b c a 1^{+/-}$, and $A b c a 1^{-/-}$mice fed normal lab chow

\begin{tabular}{lccc}
\hline Genotype & Abca1 & & \\
Bile salts $(\mu \mathrm{mol} / \mathrm{d})$ & $1.24 \pm 0.33$ & $1.38 \pm 0.30$ & $1.68 \pm 0.42$ \\
Neutral sterols $(\mu \mathrm{mol} / \mathrm{d})$ & $2.17 \pm 0.41$ & $2.31 \pm 0.20$ & $2.51 \pm 0.41$
\end{tabular}

Feces was collected from individual mice during 1 week, lyophilized, weighed, and subjected to analysis of acidic (bile salts) and neutral sterols, as outlined in Methods. Values are presented as mean \pm SD for five male animals in each group. No significant differences between any of the groups were noted. 


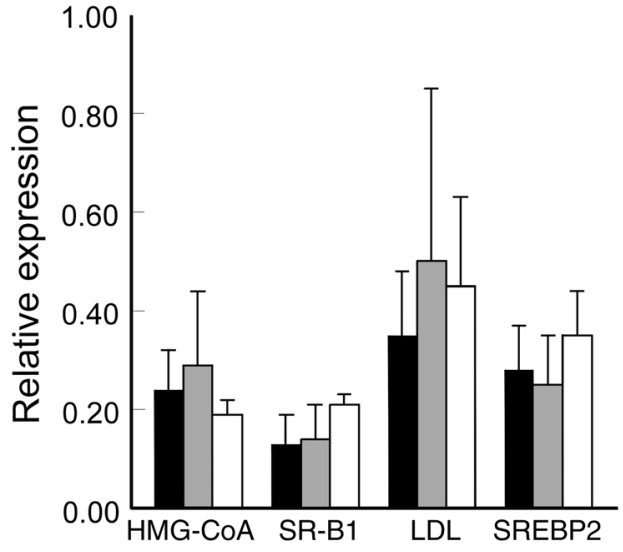

\section{Figure 2}

Expression levels of $\mathrm{HMG}-\mathrm{Co} A$ reductase, $\mathrm{LDL}$ receptor, $\mathrm{SR}-\mathrm{BI}$, and SREBP2 relative to $\beta$-actin in livers from male $\mathrm{Abca1}^{+/+}, \mathrm{Abca}^{+/-}$, and $A b c a 1^{-/-}$mice. Livers were harvested from lab chow-fed male mice, and total RNA was isolated for semiquantitative RT-PCR analysis as described in Methods. Data represent mean \pm SD for five mice in each group. Black bars, $\mathrm{Abca1^{+/+ }}$ mice; gray bars, $\mathrm{Abca1^{+/- }}$ mice; open bars, $A b c a 1^{-/-}$mice. No significant differences were noted between groups.

\section{Discussion}

In humans, plasma HDL levels are strongly correlated with the incidence of cardiovascular diseases (3). It is generally thought that defects in HDL-mediated reverse or centripetal cholesterol transport contribute to development of atherosclerotic plaques. Elucidation of the factors involved in regulation of this HDL-mediated pathway is therefore of prime importance.

Detailed information about the magnitude of the different cholesterol fluxes in the bodies of rodents has been generated in a series of elegant studies from Dietschy's group (2, 47-53). An important conclusion from their work is that reverse cholesterol transport, which they call "centripetal cholesterol flux," is not determined by the plasma level of HDL, but by processes in peripheral tissues that make cholesterol available for uptake by nascent HDL particles. Since recent work has firmly established that activity of ABCA1 is the major controlling factor for cholesterol efflux towards (nascent) HDL and maintenance of HDL levels $(31,32)$, one would expect that disruption of the gene encoding ABCA1 leads to abrogation of the bulk of centripetal flux, and hence to alterations in hepatic cholesterol metabolism and the magnitude of the transhepatic cholesterol flux. Our results, however, show unequivocally that the proposed concept of reverse cholesterol transport and the role of ABCA1 therein needs revision. Despite the absence of HDL due to inactivation of the HDL cholesterol supplier ABCA1, transhepatic cholesterol flux as measured by biliary cholesterol and bile salt secretion was unaltered in chow-fed mice as well as in mice fed a highcholesterol atherogenic diet. Accordingly, hepatic levels of cholesterol (and of phospholipids) were also unaffected by ABCA1 deficiency.
In principle, these results could be explained by assuming a compensatory increase of hepatic cholesterol synthesis to offset the loss of cholesterol supply from the periphery. However, in an earlier study with these mice, McNeish et al. (31) observed by DNA microarray analysis a decrease rather than an increase in hepatic expression of genes controlling key steps in cholesterol synthesis. In the present study, we found no differences in the expression of the genes encoding HMG-CoA reductase and the LDL receptor in livers of $A b c a 1^{-/-}, A b c a 1^{+/-}$, or wild-type mice, or in the expression of SREBP2, the key transcription factor in the regulation of cellular cholesterol homeostasis (54). Likewise, no differences in hepatic expression at the mRNA or protein (not shown) levels were found for the HDL receptor SR-BI. Clearly, unchanged gene expression does not exclude posttranscriptional/posttranslational regulation of enzyme activities. We therefore estimated in vivo the contribution to the cholesterol pool of de novo synthesis for recruitment of biliary cholesterol secretion and bile salt synthesis by MIDA. In addition, net whole-body cholesterol efflux was determined by assessing fecal neutral sterol and bile salt output. The results of both the MIDA analysis and fecal sterol measurements showed no significant differences between the three different genotypes, indicating that overall body cholesterol fluxes were not influenced by (partial) ABCA1 deficiency. Finally, the transfer of plasma-derived (liposomal) free cholesterol into the bile proceeded at a similar rate in wild-type and ABCA1-deficient mice (Figure 3).

To explain these unexpected results, one could argue that the knockout mice succeeded in fully compensating for the lack of HDL-mediated flux via upregulation of the LDL receptor/LRP pathways. Indeed, VLDL/LDL levels

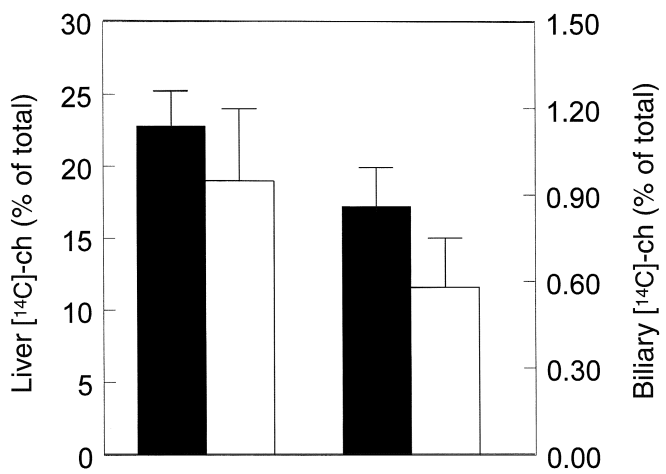

\section{Figure 3}

Liver uptake and biliary secretion of cholesterol derived from intravenously injected liposomes. Wild-type and $\mathrm{Abca1}^{-1-}$ mice were injected with small unilamellar liposomes containing $\left[{ }^{3} \mathrm{H}\right]$ cholesteryloleylether and $\left[{ }^{14} \mathrm{C}\right]$ cholesterol as described in Methods. The bile duct was cannulated and bile was collected in 30-minute intervals for 120 minutes. After 120 minutes, livers were harvested, and radioactivity in plasma, liver, and bile was measured. Data are presented for bile (open bars) as accumulated uptake in 120 minutes, expressed in percent of the injected dose, and for liver (filled bars) as percent of the injected dose at 120 minutes. Data are mean \pm SD of four male mice in each group. 


\section{Table 3}

The contribution of de novo cholesterol synthesis to biliary cholesterol and biliary cholate, determined by MIDA in $\mathrm{Abcal}^{+/+}, \mathrm{Abca}^{+/-}$, and $\mathrm{Abca}^{-/-}$mice

\begin{tabular}{|c|c|c|c|}
\hline Genotype & $A b c a 1^{+/+}$ & $A b c a 1^{+/-}$ & Abca1-/- \\
\hline$P_{\text {cholesterol }}(\%)$ & $5 \pm 0.3$ & $5.1 \pm 0.1$ & $5.5 \pm 0.2$ \\
\hline $\begin{array}{l}\text { Cholesterol fractional } \\
\text { synthesis (\%) }\end{array}$ & $26.4 \pm 1.5$ & $26.4 \pm 2.8$ & $23.6 \pm 3.3$ \\
\hline $\mathrm{P}_{\text {cholate }}(\%)$ & $4.4 \pm 0.7$ & $4.1 \pm 0.4$ & $5.3 \pm 0.2$ \\
\hline $\begin{array}{l}\text { Cholesterol fractional } \\
\text { synthesis (\%) }\end{array}$ & $12.6 \pm 3.8$ & $12.9 \pm 2.3$ & $9.7 \pm 2.6$ \\
\hline
\end{tabular}

Mice were fed rat chow containing $\left[1^{13} \mathrm{C}\right]$ acetate for 10 days prior to collection of bile. MIDA was carried out after GC/MS analysis (as described in Methods) to calculate the precursor pool enrichments $(P)$ for biliary cholesterol and for cholate, the major bile salt species in mice, respectively. The fractional contribution of newly synthesized cholesterol to biliary cholesterol and to formation of biliary cholate, representing the circulating bile salt pool, was then calculated as described (37-39). Values are presented as mean \pm SD for five male animals in each group.

are somewhat elevated in plasma of $A b c a 1^{-/-}$mice in comparison with wild-type controls (refs. 31, 32, 46, and confirmed in our study). We investigated the validity of this hypothesis by pushing this pathway to the maximum by feeding a high-cholesterol, high-cholate diet. Under such extreme conditions, compensatory mechanisms may become inadequate, and potential differences between wild-type and null mice should surface. Interestingly, even under these stressed, highly unphysiological conditions, no differences between the three genotypes were observed. Cholesterol(ester) accumulation did occur on the high-cholesterol, high-cholate diet, but it occurred to exactly the same extent in mice of all three genotypes. Most importantly, biliary secretion of bile salt and cholesterol was greatly increased by cholesterol/bile salt feeding, but again, to exactly the same extent in wild-type, heterozygous, and knockout mice.

After submission of this manuscript, a paper of Drobnik et al. (55) appeared in which body sterol homeostasis was studied in C57BL/6J Abca1null mice. The authors concluded that total body sterol homeostasis was affected because they observed a slight increase in fecal sterol output due to decreased cholesterol absorption efficiency. In accordance with our data, no differences in biliary or hepatic cholesterol concentrations were reported. Despite these unchanged cholesterol concentrations, Drobnik et al. (55) noticed a strong decrease in liver HMGCoA reductase mRNA levels and activity. The authors did not discuss the origin(s) of the extra sterol leaving the body. It should be noted that the ABCA1-deficient model with a C57BL/6J background used in these studies shows more pathology (46) than mice with a DBA/ 1 background (refs. 31, 32, and our own observations),

\section{Table 4}

which may have affected the results. According to our results, ABCA1 does not significantly control total body cholesterol fluxes, even under highly stressed conditions. Assuming that the overall centripetal cholesterol flux in the mice used in these studies is of the same order of magnitude as that reported in several other mouse models $(51,52,56)$, our results strongly suggest that ABCA1 is not involved in control of this flux. Since very low HDL levels resulting from the absence of apoA-I also do not influence centripetal cholesterol flux in mice (56-58), we hypothesize that HDL does not play a quantitatively important role in this pathway of cholesterol transport.

Studies with the SR-BI-knockout mouse seem at variance with this conclusion. In this mouse model, HDL transport is abrogated at the entry level in the liver; this leads to a $40 \%$ decrease in biliary cholesterol secretion, whereas bile salt and phospholipid secretion remain unchanged (59). At first glance, these results seem at variance with the data we obtained in the Abca1-null mouse, since HDL-mediated cholesterol transport to the liver is absent in both models. A recent study by Silver et al. (60) sheds some light on this issue. In elegant experiments, these authors provided evidence for a direct role of SR-BI in intracellular cholesterol trafficking. After uptake at the sinusoidal membrane, HDL bound to SR$\mathrm{BI}$ is partly internalized, cholesterol is delivered at the canalicular membrane, and lipid-free apoA-I shuttles back to the sinusoidal membrane, together with SR-BI. Thus, it may be that in the absence of SR-BI, intracellular cholesterol trafficking is disturbed.

Recent data of Alam et al. (61) are fully in line with the results of our study. These authors upregulated several key steps considered to be involved in the regulation of reverse cholesterol transport in mice, and did not see any effect on the net rate of reverse cholesterol transport. Although these recent findings support our hypothesis, we realize that our data do not exclude the

Plasma, biliary, and hepatic lipid values in $\mathrm{Abca}^{+/+}, \mathrm{Abca}^{+/-}$, and $\mathrm{Abca1^{-/- }}$ mice fed a high-cholesterol, high-fat, cholate-containing diet for 2 weeks

\begin{tabular}{|c|c|c|c|}
\hline Genotype & $A b c a 1^{+/+}$ & $A b c a 1^{+/-}$ & Abca1-/- \\
\hline Plasma cholesterol (mM) & $3.32 \pm 0.44$ & $2.24 \pm 0.34^{\mathrm{A}}$ & $1.34 \pm 0.51^{\mathrm{A}, \mathrm{E}}$ \\
\hline Plasma phospholipids (mM) & $2.36 \pm 0.58$ & $1.72 \pm 0.45^{\mathrm{A}}$ & $0.80 \pm 0.28^{A, E}$ \\
\hline Plasma triglycerides (mM) & $0.30 \pm .09$ & $0.29 \pm 0.04$ & $0.41 \pm 0.2$ \\
\hline Liver free cholesterol $(\mu \mathrm{mol} / \mathrm{g})$ & $11.5 \pm 1.3$ & $12.4 \pm 3.7$ & $13.2 \pm 5.7$ \\
\hline Liver cholesterol(ester) $(\mu \mathrm{mol} / \mathrm{g})$ & $48.2 \pm 11.4$ & $46.3 \pm 14.8$ & $50.5 \pm 20.1$ \\
\hline Liver phospholipids $(\mu \mathrm{mol} / \mathrm{g})$ & $31.9 \pm 2.1$ & $34.5 \pm 4.5$ & $32.1 \pm 2.3$ \\
\hline Liver triglycerides $(\mu \mathrm{mol} / \mathrm{g})$ & $22.7 \pm 2.3$ & $23.5 \pm 6.2$ & $19.4 \pm 3.2$ \\
\hline $\begin{array}{l}\text { Biliary cholesterol secretion } \\
(\mathrm{nmol} / \mathrm{min} / 100 \mathrm{~g} \mathrm{BW})\end{array}$ & $23.3 \pm 1.0$ & $27.9 \pm 2.5$ & $26.3 \pm 7.1$ \\
\hline $\begin{array}{l}\text { Biliary phospholipid secretion } \\
(\mathrm{nmol} / \mathrm{min} / 100 \mathrm{~g} \mathrm{BW})\end{array}$ & $125 \pm 29.1$ & $115 \pm 18.6$ & $124 \pm 20.4$ \\
\hline $\begin{array}{l}\text { Biliary bile salt secretion } \\
(\mathrm{nmol} / \mathrm{min} / 100 \mathrm{~g} \mathrm{BW})\end{array}$ & $903 \pm 377$ & $819 \pm 254$ & $765 \pm 238$ \\
\hline
\end{tabular}

Values are presented as mean \pm SD for six animals (four females and two males) in each group. ASignificant difference when compared with wild-type $\left(A b c a 1^{+/+}\right)$mice. BSignificant difference when compared with heterozygous $\left(\mathrm{Abca}^{+/-}\right)$mice. There was no difference between males and females. 
possibility that the loss of HDL transport capacity is compensated for by another transport mechanism. This hypothetical compensatory mechanism is not capable of preventing the development of cardiovascular disease in patients with FHD or Tangier disease, or the defects observed in both mouse strains in which the Abca1 gene has been disrupted $(23,31,32,46)$. Clearly, absence of ABCA1 activity primarily affects macrophages, and - particularly in Tangier patients this cell type is responsible for the phenotype. Macrophages require very efficient lipid handling systems to dispose of the lipids acquired during degradation of engulfed cell material and/or uptake of modified lipoproteins. ABCA1 is required for efflux of the excess cholesterol and phospholipid thus obtained. Assuming that non-macrophage-derived reverse cholesterol transport did not increase in the $\mathrm{Abca1}^{-/-}$mice, our results indicate that macrophage-derived cholesterol transport does not contribute significantly to the total magnitude of reverse cholesterol transport.

In the "classical view," it is assumed that all cholesterol synthesized in peripheral tissues eventually travels through the liver, following the old paradigm that biliary excretion is the sole pathway via which cholesterol can leave the body (2). Apart from the liver, a major cholesterol-synthesizing organ in mice is the intestine (52). It is quite conceivable that this organ also secretes cholesterol directly into its lumen, followed by fecal disposition. We have shown in a previous study that in Mdr2 P-glycoprotein-deficient mice, which lack biliary cholesterol secretion (44), fecal excretion of neutral sterols is actually doubled (42). A similar phenomenon was observed in rats in which bile was diverted for up to 2 weeks (39). With direct intestinal cholesterol secretion also being an important pathway in wild-type mice, the magnitude of the transhepatic cholesterol flux may be overestimated by up to $50 \%$. The remaining portion of the flux, however, remains unexplained at the moment. Cross-breeding of the Abca1-null mice with other knockout mouse models will have to provide the answer to this important question.

\section{Acknowledgments}

We thank Rick Havinga, Juul Baller, Jan Kamps, and Torsten Plösch for excellent technical assistance. This research was supported by grant 902-23-193 from the Netherlands Organization for Scientific Research (NWO), and by grant WS00-46 from the Maag, Lever en Darm stichting.

1. Glomset, J.A., and Norum, K.R. 1973. The metabolic role of lecithin: cholesterol acyltransferase: perspectives form pathology. Adv. Lipid. Res. 11:1-65.

2. Dietschy, J.M., Turley, S.D., and Spady, D.K. 1993. Role of liver in the maintenance of cholesterol and low density lipoprotein homeostasis in different animal species, including humans. J. Lipid. Res. 34:1637-1659.

3. Tall, A.R., and Wang, N. 2000. Tangier disease as a test of the reverse cholesterol transport hypothesis. J. Clin. Invest. 106:1205-1207.

4. Acton, S., et al. 1996. Identification of scavenger receptor sr-bi as a high density lipoprotein receptor. Science. 271:518-520.

5. Kozarsky, K.F., et al. 1997. Overexpression of the hdl receptor sr-bi alters plasma hdl and bile cholesterol levels. Nature. 387:414-417.

6. Babitt, J., et al. 1997. Murine sr-bi, a high density lipoprotein receptor that mediates selective lipid uptake, is n-glycosylated and fatty acylated and colocalizes with plasma membrane caveolae. J. Biol. Chem. 272:13242-13249.

7. Rigotti, A., et al. 1997. A targeted mutation in the murine gene encoding the high density lipoprotein (HDL) receptor scavenger receptor class B type I reveals its key role in HDL metabolism. Proc. Natl. Acad. Sci. USA. 94:12610-12615.

8. Arai, T., Wang, N., Bezouevski, M., Welch, C., and Tall, A.R. 1999. Decreased atherosclerosis in heterozygous low density lipoprotein receptor-deficient mice expressing the scavenger receptor BI transgene. J. Biol. Chem. 274:2366-2371.

9. Wang, N., Arai, T., Ji, Y., Rinninger, F., and Tall, A.R. 1998. Liver-specific overexpression of scavenger receptor BI decreases levels of very low density lipoprotein ApoB, low density lipoprotein ApoB, and high density lipoprotein in transgenic mice. J. Biol. Chem. 273:32920-32926.

10. Rothblat, G.H., et al. 1999. Cell cholesterol efflux: integration of old and new observations provides new insights. J. Lipid Res. 40:781-796.

11. Trigatti, B., Rigotti, A., and Krieger, M. 2000. The role of the high-density lipoprotein receptor SR-BI in cholesterol metabolism. Curr. Opin. Lipidol. 11:123-131.

12. Clee, S.M., et al. 2000. Age and residual cholesterol efflux affect HDL cholesterol levels and coronary artery disease in ABCA1 heterozygotes. J. Clin. Invest. 106:1263-1270.

13. Bodzioch, M., et al. 1999. The gene encoding atp-binding cassette transporter 1 is mutated in tangier disease. Nat. Gen. 22:347-351.

14. Brooks-Wilson, A., et al. 1999. Mutations in abc1 in tangier disease and familial high-density lipoprotein deficiency. Nat. Gen. 22:336-345.

15. Marcil, M., et al. 1999. Mutations in the abc1 gene in familial hdl deficiency with defective cholesterol efflux. Lancet. 354:1341-1346.

16. Drobnik, W., et al. 1999. Growth and cell cycle abnormalities of fibroblasts from tangier disease patients. Arterioscler. Thromb. Vasc. Biol. 19:28-38.

17. Eberhart, G.P., Mendez, A.J., and Freeman, M.W. 1998. Decreased cholesterol efflux from fibroblasts of a patient without Tangier disease, but with markedly reduced high density lipoprotein cholesterol levels. J. Clin. Endocrinol. Metab. 83:836-846.

18. Mendez, A.J. 1997. Cholesterol efflux mediated by apolipoproteins is an active cellular process distinct from efflux mediated by passive diffusion. J. Lipid Res. 38:1807-1821.

19. Remaley, A.T., et al. 1997. Decreased reverse cholesterol transport from Tangier disease fibroblasts. Acceptor specificity and effect of brefeldin on lipid efflux. Arterioscler. Thromb. Vasc. Biol. 17:1813-1821.

20. von Eckardstein, A., et al. 1998. Plasma and fibroblasts of Tangier disease patients are disturbed in transferring phospholipids onto apolipoprotein A-I. J. Lipid Res. 39:987-998.

21. Mott, S., et al. 2000. Decreased cellular cholesterol efflux is a common cause of familial hypoalphalipoproteinemia: role of the ABCA1 gene mutations. Atherosclerosis. 152:457-468.

22. Moynault, A., Luciani, M.F., and Chimini, G. 1998. ABC1, the mammalian homologue of the engulfment gene ced-7, is required during phagocytosis of both necrotic and apoptotic cells. Biochem. Soc. Trans. 26:629-635.

23. Hamon, Y., et al. 2000. ABC1 promotes engulfment of apoptotic cells and transbilayer redistribution of phosphatidylserine. Nat. Cell Biol. 2:399-406.

24. Wang, N., Silver, D.L., Costet, P., and Tall, A.R. 2000. Specific binding of ApoA-I, enhanced cholesterol efflux, and altered plasma membrane morphology in cells expressing ABC1. J. Biol. Chem. 275:33053-33058.

25. Chambenoit, O., et al. 2001. Specific docking of apolipoprotein A-I at the cell surface requires a functional ABCA1 transporter. J. Biol. Chem. 276:9955-9960.

26. Langmann, T., et al. 1999. Molecular cloning of the human ATP-binding cassette transporter 1 (hABC1): evidence for sterol-dependent regulation in macrophages. Biochem. Biophys. Res. Commun. 257:29-33.

27. Repa, J.J., et al. 2000. Regulation of absorption and ABC1-mediated efflux of cholesterol by RXR. Science. 289:1524-1529.

28. Robins, S.J., and Brunengraber, H. 1982. Origin of biliary cholesterol and lecithin in the rat: contribution of new synthesis and preformed hepatic stores. J. Lipid Res. 23:604-608.

29. Chanussot, F., Lafont, H., Hauton, J., Tuchweber, B., and Yousef, I. 1990. Studies on the origin of biliary phospholipid. Effect of dehydrocholic acid and cholic acid infusions on hepatic and biliary phospholipids. Biochem. J. 270:691-695.

30. Robins, S.J., and Fasulo, J.M. 1997. High density lipoproteins, but not other lipoproteins, provide a vehicle for sterol transport to bile. J. Clin. Invest. 99:380-384.

31. McNeish, J., et al. 2000. High density lipoprotein deficiency and foam cell accumulation in mice with targeted disruption of ATP-binding cassette transporter-1. Proc. Nat. Acad. Sci. USA. 97:4245-4250.

32. Orso, E., et al. 2000. Transport of lipids from golgi to plasma membrane is defective in tangier disease patients and Abc1-deficient mice. Nat. Gen. 24:192-196.

33. Jung, H.R., Turner, S.M., Neese, R.A., Young, S.G., and Hellerstein, M.K. 
1999. Metabolic adaptations to dietary fat malabsorption in chylomicron-deficient mice. Biochem. J. 343:473-478.

34. Bandsma, R.H.J., et al. 2000. The contribution of newly synthesized cholesterol to bile salt synthesis in rats quantified by mass isotopomer distribution analysis. Biochim. Biophys. Acta. 1483:343-351.

35. Kuipers, F., Spanjer, H.H., Havinga, R., Scherphof, G.L., and Vonk, R.J 1986. Lipoproteins and liposomes as in vivo cholesterol vehicles in the rat: preferential use of cholesterol carried by small unilamellar liposomes for the formation of muricholic acids. Biochim. Biophys. Acta. 876:559-566.

36. Neese, R.A., et al. 1993. Measurement of endogenous synthesis of plasma cholesterol in rats and humans using MIDA. Am. J. Physiol. 264:E136-E147.

37. Hellerstein, M.K., and Neese, R.A. 1992. Mass isotopomer distribution analysis: a technique for measuring biosynthesis and turnover of polymers. Am. J. Physiol. 263:E988-E1001.

38. Neese, R.A., et al. 1993. Measurement of endogenous synthesis of plasma cholesterol in rats and humans using mida. Am. J. Physiol. 264:E136-E147.

39. Bandsma, R.H., et al. 1998. Contribution of newly synthesized cholesterol to rat plasma and bile determined by mass isotopomer distribution analysis: bile- salt flux promotes secretion of newly synthesized cholesterol into bile. Biochem. J. 329:699-703.

40. Bloks, V.W., et al. 2001. Hyperlipidemia and atherosclerosis associated with liver disease in ferrochelatase-deficient mice. J. Lipid Res. 42:41-50.

41. Mensenkamp, A.R., et al. 1999. Apolipoprotein E participates in the regulation of very low density lipoprotein-triglyceride secretion by the liver. J. Biol. Chem. 274:35711-35718.

42. Voshol, P.J., et al. 1998. Reduced plasma cholesterol and increased fecal sterol loss in multidrug resistance gene 2 p-glycoprotein-deficient mice. Gastroenterology. 114:1024-1034.

43. Frijters, C.M., et al. 1996. Influence of bile salts on hepatic mdr2 p-glycoprotein expression. Adv. Enzyme Regul. 36:351-363.

44. Smit, J.J.M., et al. 1993. Homozygous disruption of the murine mdr2 Pglycoprotein gene leads to a complete absence of phospholipid from bile and to liver disease. Cell. 75:451-462.

45. Scherphof, G.L., Kuipers, F., Derksen, J.T., Spanjer, H.H., and Vonk, R.J. 1987. Liposomes in vivo: conversion of liposomal cholesterol to bile salts. Biochem. Soc. Trans. 15(Suppl.):62S-68S.

46. Christiansen-Weber, T.A., et al. 2000. Functional loss of ABCA1 in mice causes severe placental malformation, aberrant lipid distribution, and kidney glomerulonephritis as well as high-density lipoprotein cholesterol deficiency. Am. J. Pathol. 157:1017-1029.

47. Osono, Y., Woollett, L.A., Herz, J., and Dietschy, J.M. 1995. Role of the low density lipoprotein receptor in the flux of cholesterol through the plasma and across the tissues of the mouse. J. Clin. Invest. 95:1124-1132.
48. Jolley, C.D., Woollett, L.A., Turley, S.D., and Dietschy, J.M. 1998. Centripetal cholesterol flux to the liver is dictated by events in the peripheral organs and not by the plasma high density lipoprotein or apolipoprotein a-i concentration. J. Lipid Res. 39:2143-2149.

49. Xie, C.L., Turley, S.D., and Dietschy, J.M. 1999. Cholesterol accumulation in tissues of the niemann-pick type $\mathrm{c}$ mouse is determined by the rate of lipoprotein-cholesterol uptake through the coated-pit pathway in each organ. Proc. Natl. Acad. Sci. USA. 96:11992-11997.

50. Jolley, C.D., Dietschy, J.M., and Turley, S.D. 1999. Genetic differences in cholesterol absorption in 129/sv and c57bl/6 mice: effect on cholesterol responsiveness. Am. J. Phys. 276:G1117-G1124.

51. Xie, C., Turley, S.D., Pentchev, P.G., and Dietschy, J.M. 1999. Cholesterol balance and metabolism in mice with loss of function of niemann-pick c protein. Am. J. Physiol. 276:E336-E344.

52. Osono, Y., Woollett, L.A., Marotti, K.R., Melchior, G.W., and Dietschy, J.M. 1996. Centripetal cholesterol flux from extrahepatic organs to the liver is independent of the concentration of high density lipoproteincholesterol in plasma. Proc. Natl. Acad. Sci. USA. 93:4114-4119.

53. Dietschy, J.M. 1997. Theoretical considerations of what regulates lowdensity-lipoprotein and high-density-lipoprotein cholesterol. Am. J. Clin. Nutr. 65(Suppl. 5):1581S-1589S.

54. Brown, M.S., and Goldstein, J.L. 1997. The SREBP pathway: regulation of cholesterol metabolism by proteolysis of a membrane-bound transcription factor. Cell. 89:331-340.

55. Drobnik, W., et al. 2001. ATP-binding cassette transporter A1 (ABCA1) affects total body sterol metabolism. Gastroenterology. 120:1203-1211.

56. Jolley, C.D., Dietschy, J.M., and Turley, S.D. 2000. Induction of bile acid synthesis by cholesterol and cholestyramine feeding is unimpaired in mice deficient in apolipoprotein AI. Hepatology. 32:1309-1316.

57. Jian, B., et al. 1998. Scavenger receptor class B type I as a mediator of cellular cholesterol efflux to lipoproteins and phospholipid acceptors. $J$. Biol. Chem. 273:5599-5606.

58. Ji, Y., et al. 1999. Hepatic scavenger receptor BI promotes rapid clearance of high density lipoprotein free cholesterol and its transport into bile.J. Biol. Chem. 274:33398-33402.

59. Mardones, P., et al. 2001. Hepatic cholesterol and bile acid metabolism and intestinal cholesterol absorption in scavenger receptor class B type I-deficient mice. J. Lipid Res. 42:170-180.

60. Silver, D.L., Nan, W., Xiao, X., and Tall, A.R. 2001. HDL particle uptake mediated by SR-BI results in selective sorting of HDL cholesterol from protein and polarized cholesterol secretion. J. Biol. Chem. 276:25287-25293.

61. Alam, K., Meidell, R.S., and Spady, D.K. 2001 Effect of up-regulating individual steps in the reverse cholesterol transport pathway on reverse cholesterol transport in normolipidemic mice. J. Biol. Chem. 276:15641-15649. 